\section{IDEAL FOR MINIMAL INVASIVE FLAPLESS SURGERY}

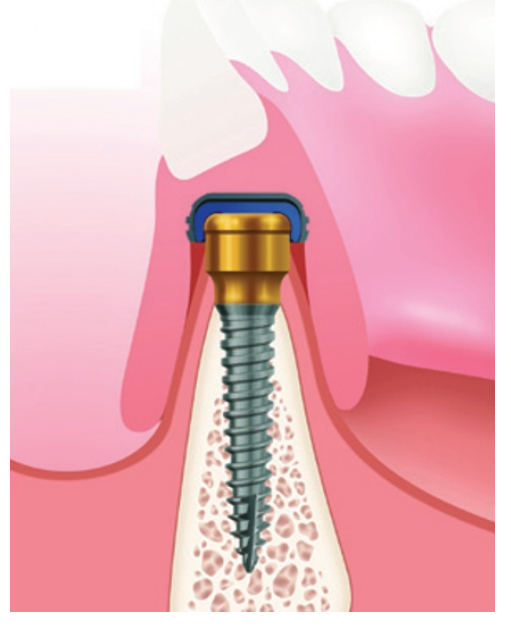

The LODI Locator Overdenture Implant System from Zest can be used to securely retain both maxillary and mandibular overdentures and is ideal for cases with narrow ridges, where there are financial considerations or when the operator wants minimal invasive flapless surgery. Costing just £112.12 plus VAT each they are genuine practice builders and revenue generators.

Ready for immediate placement, self-tapping LODI implants are available in two diameters and three lengths together with Zest Locator Abutments with a choice of two soft tissue cuff heights: 12 options in total.

Manufactured from the strongest titanium alloy available, LODI implants have a proven RBM surface with a $1.5 \mu$ texture, which increases primary stability and bone to implant contact, thereby improving bone anchoring. The Zest Locator attachments incorporate all of Locator's unique features including its patented pivoting technology and customisable retention levels, while maintaining a dramatically reduced vertical height compared with 0-ball mini implants.

They are supplied in sterile packaging containing all the essential components for use (implant, attachment and processing pack).

For further information call 01380734990 or visit www.generalmedical.co.uk.

\title{
SIMULTANEOUS SURGICAL AND PROSTHETIC PLANNING
}

Sirona Dental Systems has introduced ground-breaking solutions for integrated implant planning and treatment, solutions that combine the digital impression scanning and design capabilities of CEREC with the diagnostic accuracy of Sirona's 3D X-ray units. Combine CEREC digital impressions and computer-aided design with the diagnostic security of Sirona's 3D X-ray systems for genuine integrated implantology. It is now possible to perform surgical and prosthetic planning simultaneously - and then implement this plan precisely using a surgical guide.

This is ideal for all practitioners who would like to place more implants in future - or those who want to provide implant therapy for the first time with higher clinical security as a result of the surgical guides and perfect documentation of the implant job. You save time and money with fewer treatment sessions and achieve significant efficiency gains with a streamlined workflow. You can also show your patients what the final result will look like, leading to higher treatment plan acceptance.

For further information call Sirona Dental Systems on 08450715040 or email info@sironadental.co.uk.

\section{EASY TO HANDLE; NOT STICKY}

$\mathrm{X}$-Glass is a remarkably effective, resin-free, biocompatible and radiopaque glass ionomer from UnoDent.

UnoDent X-Glass is easy to handle and non-sticky so placing the perfect restoration is simple. It's also fast setting, which means that patient chair time is significantly shortened.

From January to February 2014, products from the UnoDent X-Glass range are available from The Dental Directory at an incredible 25\% off of their regular price.

Grab hold of these great savings and refill your glass ionomer inventory for the New Year. Place

\section{ENHANCING BONE DEVELOPMENT}

Expand your restorative options and achieve beautiful, healthier outcomes with the NobelReplace Conical Connection implant system from Nobel Biocare. The conical connection fastens the implants to abutments so tightly they significantly reduce micro-movement and the micro-gap to enhance bone development and soft tissue.

NobelReplace Conical Connection ensures high primary stability in all indications for reliable, long lasting results. The strong and tight conical connection keeps bacteria away from the connection area that could otherwise contribute towards inflammation, tissue recession and bone loss.

Clinicians are safe in the knowledge they are doing all they can for their patients to ensure the long-term success of their implant treatment. They are also guaranteed peerless customer service and support.

For more information, contact Nobel Biocare on 0208756 3300, or visit www.nobelbiocare.com. your orders for UnoDent X-Glass from The Dental Directory today. www.dental-directory.co.uk

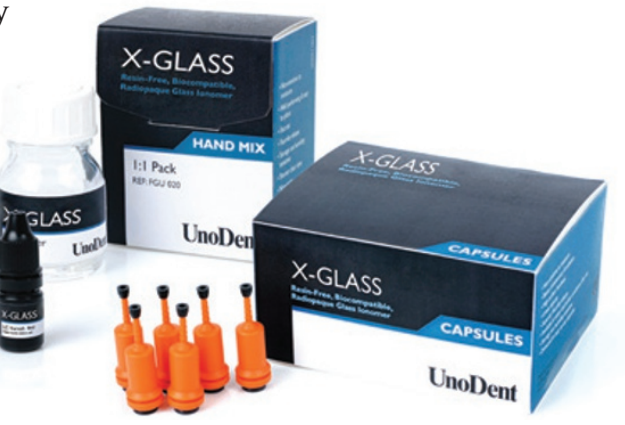

\section{THE FIRST BULK-FILL COMPOSITE}

Faster treatment time is just one of the many advantages of SDR (Smart Dentine Replacement), the first bulk-fill composite material from DENTSPLY.

SDR allows you to fill at convenient $4 \mathrm{~mm}$ increments, making restoration work much faster and easier compared to traditional composites which require cumbersome layering techniques.

SDR's technological advancements allow this bulk-fill restorative with self-levelling properties to provide high quality restorations that have been proven to perform excellently in three-year clinical trials.

For further information see DENTSPLY's website at www. dentsply.co.uk or telephone 0800 0723313. 